\title{
Fatores de risco para injúrias acidentais em pré-escolares
}

\author{
Risk factors for accidental injuries in preschool children \\ Sílvia S. Fonseca ${ }^{1}$, Cesar G. Victora ${ }^{2}$, Ricardo Halpern ${ }^{3}$, Aluísio J.D. Barros ${ }^{4}$, \\ Rosângela C. Lima ${ }^{5}$, Luciane A. Monteiro ${ }^{6}$, Fernando Barros ${ }^{7}$
}

\section{Resumo}

Objetivo: medir os principais fatores de risco relacionados à ocorrência de injúrias acidentais, na faixa etária entre quatro e cinco anos de idade.

Métodos: foi estudada uma coorte prospectiva de 620 crianças, na qual a ocorrência de acidentes e injúrias foi registrada em um diário, durante um período de um mês. Esta foi uma subamostra sistemática, proveniente da coorte de nascimentos de 1993, que ainda residiam na área urbana de Pelotas, RS. O desfecho em estudo foi o número de injúrias acidentais relatadas por criança-mês. A análise multivariada, utilizando Regressão de Poisson, foi usada para controlar fatores de confusão.

Resultados: a incidência mensal de acidentes foi de $53,8 \%$, e $48,4 \%$ das crianças sofreram pelo menos uma injúria acidental. As crianças do sexo masculino tiveram $30 \%$ mais de chances de se lesionarem do que aquelas do sexo feminino, e as crianças brancas tiveram um risco $70 \%$ maior do que as crianças não-brancas. Renda familiar, escolaridade dos pais e trabalho materno não se associaram à ocorrência de injúrias. Após ajuste para variáveis socioeconômicas e ambientais, as crianças que possuíam um ou mais irmãos menores apresentavam taxa $30 \%$ maior de injúrias acidentais. Crianças residentes em casa de tijolo apresentaram uma incidência de injúrias cerca de $40 \%$ superior.

Conclusões: poucos fatores de risco modificáveis foram associados a um aumento na freqüência de injúrias acidentais. Destes fatores, a presença de crianças mais jovens em casa merece especial atenção, sendo necessário instruir os pais sobre o aumento no risco observado por ocasião do nascimento de um irmão menor.

J Pediatr (Rio J) 2002; 78 (2):97-104: acidentes, injúrias físicas, prevenção de acidentes, fatores de risco.

1. Mestre em Epidemiologia. Médica Pediatra do Departamento MaternoInfantil da Universidade Federal de Pelotas(UFPEL), RS.

2. Doutor em Epidemiologia. Prof. Titular do Departamento de Medicina Social da UFPEL.

3. Doutor em Pediatria. Prof. do Departamento Infantil da FUCMPA.

4. Doutor em Epidemiologia. Prof. Adjunto do Departamento de Medicina Social da UFPEL.

5. Aluna do Doutorado de Epidemiologia da UFPEL.

6. Aluna da graduação da Faculdade de Medicina da UFPEL.

7. Doutor em Epidemiologia. Pesquisador do Centro Latino-americano de Perinatologia, Montevideo, Uruguai.

Artigo submetido em 31.05.01, aceito em 31.10.01.

\begin{abstract}
Objective: to measure the main risk factors associated with the occurrence of accidental injuries in children aged 4-5 years.

Methods: the study included a prospective cohort of children whose parents filled in a diary recording accidents and injuries during a period of one-month. The children represented a systematic subsample from a population-based birth cohort in southern Brazil. The outcome was the number of reported injuries per child during one month. Multivariate analysis (Poisson regression) was used to assess confounding factors.

Results: the monthly frequency of accidents was $53.8 \%$, and $48.4 \%$ of the children suffered at least one injury. Boys had $30 \%$ more injuries than girls, and white children had $70 \%$ higher incidence than non-white. Family income, parental education and maternal employment were not associated with the frequency of injuries. After adjustment of socioeconomic and environmental factors, having younger siblings was associated with a $30 \%$ higher injury rate, and living in a home made of bricks was associated with a $35 \%$ increase. The incidence of injuries appeared to be higher among children attending day-care centers and those living in periurban areas.

Conclusions: few risk factors were associated with an increased frequency of injuries. Among them, the most amenable to intervention seems to be the presence of younger siblings. Parents should become aware of children's needs for increased attention when a younger sibling is born.
\end{abstract}

J Pediatr (Rio J) 2002; 78 (2):97-104: accidents, injury, injury prevention, risk factors.

\section{Introdução}

Os acidentes na infância, apesar de potencialmente previsíveis e preveníveis, causam alta morbidade e mortalidade $^{1,2}$.

Os estudos sobre fatores de risco para ocorrência de acidentes são, em sua maioria, retrospectivos, geralmente baseados em registros de serviços de saúde ou informações colhidas através de inquéritos. Tal estratégia pode levar a 
um sub-registro importante da real incidência dos acidentes ${ }^{3}$. Os estudos prospectivos, descritos na literatura, baseiam-se geralmente em acidentes que tiveram atendimento médico, omitindo, portanto, informações a respeito de lesões menos graves. Em trabalho recente ${ }^{4}$, observou-se que o registro prospectivo de acidentes feito pela família apresenta importantes vantagens sobre os métodos acima.

Sexo masculino, baixo nível socioeconômico, presença de dois ou mais irmãos e cuidado da criança pela mãe, em alguns estudos em países desenvolvidos ${ }^{5,6} \mathrm{e}$ em desenvolvimento $^{7-9}$, têm sido apontados como alguns dos principais fatores de risco para injúrias acidentais. Outros estudos, porém, não encontraram esta relação ${ }^{10,11}$. Foi possível localizar apenas dois estudos brasileiros sobre fatores de risco para acidentes na infância. Unglert et al. ${ }^{7}$ realizaram um estudo em crianças que freqüentavam dois postos de assistência médica de bairros situados na periferia de São Paulo. Observaram que as famílias "com acidentes" apresentavam pais e mães mais velhos, maior número de filhos e pequena proporção de filhos com idades menores. Teixei$\mathrm{ra}^{10}$ realizou um estudo transversal, de base populacional, com crianças de zero a 10 anos, investigando acidentes que exigiam assistência médica. Os fatores de risco para acidentes foram sexo masculino, aumento da idade, famílias com melhor nível de escolaridade e disposição de mais bens de consumo, como os principais fatores de risco para esses acidentes. Frente a esses dados conflitantes, torna-se imperativo a realização de novos estudos que avaliem os reais fatores de risco para injúrias acidentais.

Este é o primeiro estudo prospectivo realizado no Brasil e que teve como objetivo medir os principais fatores de risco, relacionados à ocorrência de injúrias acidentais.

\section{Material e métodos}

A metodologia utilizada foi descrita detalhadamente em artigo anterior ${ }^{4,12}$. Todos os 5.304 nascimentos hospitalares ocorridos em Pelotas, RS, no ano de 1993, foram incluídos, dando origem à amostra estudada. Subamostras das crianças desta coorte foram acompanhadas no $1^{\circ}, 3^{\circ}, 6^{\circ}$ e $12^{\circ}$ meses de idade. Para as visitas de um e três meses, foi realizada amostragem sistemática de 655 crianças. Com seis e 12 meses, 1.460 foram selecionadas, representando uma amostragem sistemática de $20 \%$ de toda a coorte, incluindo as 655 acima referidas, além de todas aquelas com peso de nascimento inferior a 2.500 gramas. Destas 1.460 crianças, $1.414(96,8 \%)$ foram localizadas aos seis meses de idade, e $1.363(93,4 \%)$ aos 12 meses.

O presente estudo realizou-se no período de novembro de 1997 a maio de 1998. Das 1.363 crianças visitadas aos 12 meses de idade, selecionou-se, sistematicamente, uma amostra de 680, com base na data de nascimento. Após a aplicação de um questionário padronizado e pré-codificado por sete entrevistadoras previamente treinadas, à mãe ou ao responsável era solicitado registrar a ocorrência de acidentes em um formulário (diário de acidentes). Este era acom- panhado de uma carta explicativa aos pais, informando qual a maneira correta de preenchê-lo. Todas aquelas situações em que a criança sofresse injúria física, ou que ela poderia tê-las sofrido, deveriam ser registradas. A entrevistadora informava o preenchimento do diário e, ainda que após um período de 30 dias, ele seria recolhido. Através desse formulário, foi possível obter-se informações a respeito da ocorrência e do tipo de acidente, da lesão, quando houvessem, do turno em que ocorreu, da pessoa que estava junto à criança e da conduta adotada. Posteriormente os autores classificavam a injúria física em leve, moderada ou grave, conforme a conduta adotada, respectivamente: autotratamento em casa, consulta médica e hospitalização e/ou morte.

Foi utilizado como desfecho, entretanto, o número de acidentes em que a criança sofreu injúria física. Utilizaramse diversas variáveis como possíveis fatores de risco para a ocorrência de injúrias. Dada a natureza do estudo de coorte, as variáveis foram colhidas através dos questionários realizados em diferentes idades e na listagem abaixo, estão assim assinaladas: $\mathrm{N}$ (logo após o nascimento); $12 \mathrm{~m}$ (aos 12 meses) e $54 \mathrm{~m}$ (as colhidas na última visita).

Variáveis socioeconômicas: renda familiar total no último mês $(54 \mathrm{~m})$, escolaridade do pai $(54 \mathrm{~m})$, escolaridade materna (54m), trabalho materno - tipo e ocupação (54m) e classe social -ABIPEME (54m).

Variáveis biológicas: sexo $(54 \mathrm{~m})$ e cor observados pelas entrevistadoras (54m).

Variáveis reprodutivas: idade da mãe $(54 \mathrm{~m})$ e paridade (54m).

Variáveis ambientais: número de pessoas na casa e que dormem com a criança $(54 \mathrm{~m})$, presença de outra criança na casa (54m), tipo de construção (54m) e local de moradia centro ou periferia $(54 \mathrm{~m})$.

Variáveis perinatais: peso de nascimento $(\mathrm{N})$ e idade gestacional - através do teste de Dubowitz ${ }^{13}(\mathrm{~N})$.

Características infantis: suspeita de atraso no desenvolvimento neuropsicomotor da criança - através do teste de Denver II ${ }^{14}$ aos 12 meses (12m) e duração da amamentação (12m).

Cuidado da criança: através da informação de quem normalmente tomava conta da criança $(54 \mathrm{~m})$ e de sua frequiência à creche e/ou escola $(54 \mathrm{~m})$.

A análise estatística incluiu o teste de qui-quadrado, para as tabelas de contingência, e teste de tendência linear em proporções. Calcularam-se as razões de prevalências para a ocorrência de uma ou mais lesões e seus intervalos de confiança de $95 \%$. Calculou-se também a razão de densidades de incidências (RDI) para o número de ocorrência de injúrias acidentais e seus intervalos de confiança de $95 \%$. $\mathrm{Na}$ análise dos dados, efetuou-se um procedimento de ponderação, a fim de reproduzir a coorte original em relação ao peso de nascimento. Foram utilizados os programas SPSS for Windows e Stata 5.0. Para entrada de dados, utilizou-se o programa EPI-Info 6.0. 
A análise multivariada foi realizada conforme o modelo hierárquico definido a priori (Figura 1) ${ }^{15}$. No primeiro nível, foram incluídas as variáveis socioeconômicas e biológicas (primeiro nível hierárquico), mesmo aquelas não significativas na análise bivariada. As variáveis foram conservadas no modelo, como potenciais fatores de confusão, e entraram no ajuste do próximo nível, no caso, as reprodutivas e as ambientais (segundo nível hierárquico). $\mathrm{O}$ terceiro nível incluiu as variáveis perinatais, e o quarto nível, as características infantis e atenção à criança. As variáveis selecionadas em um determinado nível permaneceram nos modelos subseqüentes e foram consideradas como fatores de risco para a ocorrência de injúrias acidentais, mesmo que, com a inclusão daquelas hierarquicamente inferiores, houvessem perdido sua significância.

\begin{tabular}{|c|c|c|}
\hline 1 & Fatores socioeconômicos & Fatores biológicos \\
\hline 2 & Fatores reprodutivos & Fatores ambientais \\
\hline 3 & Prematuridade & Peso de nascimento \\
\hline 4 & $\begin{array}{l}\text { Cuidado da criança } \\
\text { Amamentação }\end{array}$ & Desenvolvimento \\
\hline \multicolumn{3}{|c|}{ Ocorrência de injúrias acidentais } \\
\hline
\end{tabular}

Figura 1 - Modelo hierárquico dos fatores de risco para ocorrência de injúrias acidentais

Foi utilizada a regressão de Poisson, em função de o desfecho ser uma contagem do número de injúrias acidentais. Estima-se que haja diferenças entre as crianças que sofreram uma lesão e aquelas que sofreram duas ou mais injúrias acidentais, sendo este dado observado pela regressão de Poisson ${ }^{16}$. A inclusão/exclusão de variáveis foi definida através do teste de Wald ${ }^{17}$. A adequação dos dados à distribuição de Poisson foi avaliada pela comparação do desvio (deviance) com seu número de graus de liberdade. Quando observado um desvio muito superior aos graus de liberdade (super dispersão) ${ }^{16,17}$, o parâmetro de escala do modelo foi corrigido, de forma que as variâncias dos coeficientes fossem estimadas adequadamente.

O projeto de pesquisa no qual foi inserido este trabalho foi apresentado ao Comitê Nacional de Ética em pesquisa, tendo sido aprovado.

\section{Resultados}

Das 680 famílias selecionadas, $620(91,2 \%)$ completaram o diário de acidentes. A incidência destes nesta amostra foi de $53,8 \%$, sendo que $48,4 \%$ sofreram pelo menos uma injúria física.

A Tabela 1 mostra a distribuição do número de injúrias sofridas pelas crianças em um mês. Quase metade das crianças lesionadas sofreu apenas uma lesão $(22,7 \%)$.

Além da distribuição percentual da amostra nas diferentes categorias de risco, a Tabela 2 mostra a frequiência de lesões em cada categoria, a razão de prevalências para haver sofrido uma ou mais lesões e a razão de densidades de incidências (RDI) (considerando que uma criança pode ter sofrido mais de uma lesão no período). Por ser esta última medida a de maior relevância epidemiológica, a discussão abaixo está centrada nesses resultados.

A incidência de injúrias acidentais foi maior no sexo masculino, tendo os meninos apresentado uma taxa $40 \%$ maior. Crianças brancas tiveram incidência maior $(\mathrm{RDI}=1,70)$ do que as crianças não-brancas. Classe social, renda familiar, escolaridade dos pais e trabalho materno não se encontravam associados ao relato de lesões (Tabela 2).

Os fatores reprodutivos não se encontraram associados ao desfecho. Houve tendência a maior incidência de lesões, quando a mãe tinha quatro filhos ou mais, mas a associação ficou no limiar da significância estatística $(\mathrm{p}=0,06)$ (Tabela 2).

As variáveis ambientais, descritas na Tabela 3, tais como construção de tijolo, moradia na periferia e presença de irmão menor na casa, encontravam-se positivamente associadas às injúrias acidentais.

Crianças com peso de nascimento superior a 2.500 gramas pareceram apresentar incidência maior de injúrias acidentais $(\mathrm{RDI}=1,43)$, mas essa associação não alcançou significância estatística $(\mathrm{p}=0,07)$. Não se demonstrou associação entre prematuridade e injúrias acidentais (Tabela 3).

Não se associaram ocorrência de injúrias acidentais com aleitamento materno e escore de desenvolvimento. Crianças que freqüentavam escolas ou creches não apresentaram, na análise bivariada, incidência maior de injúrias acidentais (Tabela 3).

Tabela 1 - Distribuição das crianças conforme o número de acidentes com lesão no mês, Pelotas, 1998

\begin{tabular}{ccc}
\hline $\mathbf{N}^{\mathbf{0}}$ de injúrias acidentais & $\mathbf{N}^{\mathbf{0}}$ de crianças & $\mathbf{( \% )}$ \\
\hline 0 & 320 & $(51,6)$ \\
1 & 141 & $(22,7)$ \\
2 & 85 & $(13,7)$ \\
3 & 47 & $(7,6)$ \\
4 & 18 & $(2,9)$ \\
5 & 4 & $(0,6)$ \\
6 & 3 & $(0,5)$ \\
7 & 1 & $(0,2)$ \\
8 & 1 & $(0,2)$ \\
\hline Total & 620 & $(100)$ \\
\hline
\end{tabular}


Tabela 2 - Freqüência de injúrias acidentais, conforme variáveis demográficas, socioeconômicas e reprodutivas, Pelotas, 1999

\begin{tabular}{|c|c|c|c|c|c|}
\hline Variável & Freqüência (\%) & $\begin{array}{l}\text { Freqüiência de injúrias } \\
\text { acidentais }(\%)^{\mathrm{a}}\end{array}$ & $\begin{array}{c}\text { Razão de Prevalências } \\
\text { (IC 95\%) }\end{array}$ & $\begin{array}{c}\text { Razão de Densidade } \\
\text { de Incidências (IC 95\%) }\end{array}$ & p-valor* \\
\hline \multicolumn{6}{|l|}{ Sexo } \\
\hline feminino & $329(53,1)$ & $114(34,7)$ & 1,0 & 1,0 & 0,002 \\
\hline masculino & $291(46,9)$ & $117(40,2)$ & $1,16(0,95-1,42)$ & $1,39(1,13-1,70)$ & \\
\hline \multicolumn{6}{|l|}{ Cor } \\
\hline não branca & $146(23,5)$ & $60(33,4)$ & 1,0 & 1,0 & $<0,001$ \\
\hline branca & $474(76,5)$ & $171(39,6)$ & $1,14(0,91-1,43)$ & $1,70(1,27-2,25)$ & \\
\hline \multicolumn{6}{|l|}{ Classe social $^{\text {II }}$} \\
\hline \multicolumn{6}{|l|}{ ABIPEME } \\
\hline$A+B$ & $82(13,4)$ & $30(37,0)$ & 1,0 & 1,0 & 0,47 \\
\hline $\mathrm{C}$ & $193(31,4)$ & $69(35,9)$ & $0,98(0,69-1,38)$ & $0,95(0,68-1,31)$ & \\
\hline $\mathrm{D}$ & $235(38,3)$ & $94(39,8)$ & $1,09(0,79-1,51)$ & $0,84(0,61-1,16)$ & \\
\hline $\mathrm{E}$ & $104(16,9)$ & $41(39,8)$ & $1,08(0,74-1,56)$ & $1,04(0,73-1,49)$ & \\
\hline \multicolumn{6}{|c|}{$\begin{array}{l}\text { Renda familiar II } \\
\text { (salário-mínimo) }\end{array}$} \\
\hline$>6$ & $147(23,9)$ & $50(34,1)$ & 1,0 & 1,0 & 0,85 \\
\hline $3,01-6,0$ & $165(26,8)$ & $60(36,4)$ & $1,07 \quad(0,79-1,45)$ & $0,77(0,58-1,06)$ & \\
\hline $1,01-3,0$ & $213(34,6)$ & $95(44,8)$ & $1,31(1,0-1,72)$ & $1,09(0,85-1,42)$ & \\
\hline$<1$ & $91(14,7)$ & $30(32,6)$ & $0,97(0,67-1,40)$ & $0,78(0,55-1,12)$ & \\
\hline \multicolumn{6}{|c|}{$\begin{array}{l}\text { Escolaridade materna } \\
(\operatorname{anos}) \mathbb{I}\end{array}$} \\
\hline$\geq 9$ & $156(25,3)$ & $58(37,1)$ & 1,0 & 1,0 & 0,23 \\
\hline$\overline{5}-9$ & $306(49,8)$ & $118(38,5)$ & $1,04(0,81-1,33)$ & $0,80(0,64-1,04)$ & \\
\hline $0-4$ & $153(24,9)$ & $59(38,8)$ & $1,04(0,78-1,38)$ & $0,82(0,70-1,22)$ & \\
\hline \multicolumn{6}{|c|}{$\begin{array}{l}\text { Escolaridade paterna } \\
(\text { anos) } \mathbb{T}\end{array}$} \\
\hline$\geq 9$ & $151(24,6)$ & $55(36,6)$ & 1,0 & 1,0 & 0,49 \\
\hline$\overline{5-8}$ & $307(50,0)$ & $119(38,9)$ & $1,06(0,83-1,37)$ & $0,81(0,64-1,04)$ & \\
\hline $0-4$ & $156(25,4)$ & $60(38,3)$ & $1,06(0,79-1,41)$ & $0,93(0,70-1,22)$ & \\
\hline \multicolumn{6}{|c|}{ Trabalho materno ${ }^{\mathrm{TI}}$} \\
\hline não & $295(47,7)$ & $118(40,0)$ & 1,0 & 1,0 & 0,32 \\
\hline $\operatorname{sim}$ & $323(52,3)$ & $112(34,7)$ & $0,87(0,71-1,06)$ & $0,90(0,73-1,11)$ & \\
\hline \multicolumn{6}{|c|}{ Idade materna (anos) } \\
\hline$<20$ & $111(17,9)$ & $52(46,8)$ & 1,0 & 1,0 & 0,15 \\
\hline $20-29$ & $335(54,0)$ & $124(37,0)$ & $0,79(0,62-1,101)$ & $0,80(0,65-1,14)$ & \\
\hline $30-35$ & $112(18,1)$ & $39(34,8)$ & $0,74(0,54-1,03)$ & $0,95(0,67-1,34)$ & \\
\hline$>35$ & $62(10,0)$ & $16(25,8)$ & $0,55(0,35-0,88)$ & $0,67(0,41-1,02)$ & \\
\hline \multicolumn{6}{|l|}{ Paridade } \\
\hline$<4$ & $57(9,2)$ & $19(33,3)$ & 1,0 & 1,0 & 0,06 \\
\hline$\geq 4$ & $563(90,8)$ & $212(37,7)$ & $1,23(0,82-1,85)$ & $1,51(0,98-2,32)$ & \\
\hline
\end{tabular}

* $\chi^{2}$ de Pearson † Relativa à ocorrência de uma ou mais injúrias acidentais

A análise multivariada, utilizando regressão de Poisson, foi usada para avaliar fatores de confusão. A Tabela 4 mostra as razões de densidades de incidências (RDI) brutas, para cada variável, e aquelas ajustadas para as outras variáveis no modelo hierarquizado. Todas as variáveis, significativas ou não, na análise bruta, foram levadas à análise multivariada.

No primeiro nível hierárquico, as variáveis cor da criança e sexo permaneceram altamente associadas. No
‡ Análise através de regressão de Poisson "Dados apresentam "missing"

segundo nível, construção de tijolos e presença de irmão menor na casa tiveram associação significativa com maior incidência de injúrias acidentais. Na análise bruta, moradia na periferia encontrava-se positivamente associada ao desfecho, mas, após o ajuste, esta associação mostrou-se limítrofe $(\mathrm{p}=0,07)$.

No terceiro nível, crianças com peso de nascimento superior a 2.500 gramas apresentavam uma RDI de 1,46, mas novamente a associação esteve no limiar da significância $(\mathrm{p}=0,07)$. 
Tabela 3 - Freqüência de injúrias acidentais, conforme características ambientais, do recém-nascido, da criança e cuidados com a criança, Pelotas, 1998

\begin{tabular}{|c|c|c|c|c|c|}
\hline Variável & Freqüência (\%) & $\begin{array}{l}\text { Freqüiência de injúrias } \\
\text { acidentais }(\%)^{\dagger}\end{array}$ & $\begin{array}{l}\text { Razão de Prevalências } \\
(\text { IC } 95 \%)^{\text {a }}\end{array}$ & $\begin{array}{c}\text { Razão de Densidade } \\
\text { de Incidências (IC 95\%) }\end{array}$ & p-valor* \\
\hline \multicolumn{6}{|c|}{ Aglomeração ${ }^{\mathbb{I I}}$ (pessoas) } \\
\hline $2-3$ & $357(58,0)$ & $134(37,4)$ & 1,0 & 1,0 & 0,61 \\
\hline $4-5$ & $194(31,5)$ & $75(38,5)$ & $1,03(0,82-1,29)$ & $0,83(0,66-1,05)$ & \\
\hline$>5$ & $65(10,5)$ & $27(41,3)$ & $1,11(0,81-1,52)$ & $1,14(0,81-1,60)$ & \\
\hline \multicolumn{6}{|l|}{ Tipo de casa } \\
\hline outros materiais & $116(18,7)$ & $38(32,8)$ & 1,0 & 1,0 & 0,04 \\
\hline tijolo & $504(81,3)$ & $193(38,3)$ & $1,17(0,88-1,55)$ & $1,36(1,02-1,82)$ & \\
\hline \multicolumn{6}{|l|}{ Mora no centro } \\
\hline $\operatorname{sim}$ & $558(90,0)$ & $198(35,5)$ & 1,0 & 1,0 & 0,03 \\
\hline não & $62(10,0)$ & $33(53,2)$ & $1,50(1,16-1,94)$ & $1,37(1,11-1,80)$ & \\
\hline \multicolumn{6}{|c|}{ Irmão menor na casa } \\
\hline não & $424(68,4)$ & $159(37,5)$ & 1,0 & 1,0 & 0,03 \\
\hline $\operatorname{sim}$ & $196(31,6)$ & $72(36,7)$ & $0,98(0,79-1,22)$ & $1,26(1,02-1,55)$ & \\
\hline \multicolumn{6}{|c|}{ Irmão maior na casa } \\
\hline não & $286(46,1)$ & $116(40,6)$ & 1,0 & 1,0 & \\
\hline $\operatorname{sim}$ & $334(53,9)$ & $115(34,4)$ & $0,85(0,69-1,04)$ & $0,91(0,75-1,13)$ & 0,39 \\
\hline \multicolumn{6}{|l|}{ Peso de nascimento' } \\
\hline$<2500$ & $62(10,1)$ & $21(34,0)$ & 1,0 & 1,0 & \\
\hline$\geq 2500$ & $553(89,9)$ & $214(38,7)$ & $1,14(0,79-1,64)$ & $1,43(0,97-2,12)$ & 0,07 \\
\hline \multicolumn{6}{|c|}{ Idade gestacional II (Dubowitz) } \\
\hline$\geq 37$ & $559(91,0)$ & $210(37,5)$ & 1,0 & 1,0 & 0,45 \\
\hline$<37$ & $56(9,0)$ & $25(45,6)$ & $1,21(0,89-1,65)$ & $1,14(0,81-1,62)$ & \\
\hline \multicolumn{6}{|c|}{ Teste de triagem de desenvolvimento ${ }^{\mathbb{I}}$} \\
\hline negativo & $371(60,0)$ & $145(39,1)$ & 1,0 & 1,0 & \\
\hline positivo & $247(40,0)$ & $85(34,4)$ & $0,88(0,77-1,09)$ & $0,87(0,70-1,08)$ & 0,21 \\
\hline \multicolumn{6}{|c|}{ Duração da amamentação ${ }^{\mathbb{I} i}$} \\
\hline nunca mamou & $39(6,3)$ & $18(46,2)$ & 1,0 & 1,0 & 0,29 \\
\hline$<3 \mathrm{~m}$ & $280(45,5)$ & $106(37,9)$ & $0,86(0,56-1,31)$ & $1,20(0,79-1,80)$ & \\
\hline 3 a $6 \mathrm{~m}$ & $94(15,3)$ & $33(35,1)$ & $0,76(0,47-1,23)$ & $1,02(0,96-2,28)$ & \\
\hline 6 a $12 \mathrm{~m}$ & $84(13,7)$ & $27(32,1)$ & $0,70(0,43-1,15)$ & $1,47(0,96-2,28)$ & \\
\hline$>12 \mathrm{~m}$ & $118(19,2)$ & $46(39,0)$ & $0,84(0,53-1,32)$ & $1,12(0,73-1,73)$ & \\
\hline \multicolumn{6}{|c|}{ Freqüenta creche ou escola } \\
\hline não & $513(82,7)$ & $186(36,3)$ & 1,0 & 1,0 & 0,33 \\
\hline $\operatorname{sim}$ & $107(17,3)$ & $45(42,1)$ & $1,16(0,90-1,49)$ & $1,14(0,88-1,46)$ & \\
\hline \multicolumn{6}{|c|}{ Quem cuida da criança } \\
\hline outro & $263(42,4)$ & $86(32,2)$ & 1,0 & 1,0 & 0,18 \\
\hline mãe ou pai & $357(57,6)$ & $145(42,8)$ & $1,24(1,02-1,54)$ & $1,15(0,93-1,42)$ & \\
\hline
\end{tabular}

Finalmente, duas variáveis do quarto nível hierárquico apresentavam também associações limítrofes: crianças cuidadas pelos pais $(\mathrm{p}=0,07)$, e que freqüentam creche ou escola $(p=0,10)$.

\section{Discussão}

O desfecho medido nesse estudo prospectivo foi o número de episódios observados durante o período de seguimento, ou seja, a densidade de incidência ${ }^{16}$. Foi utilizada, portanto, como método analítico dos dados, a regressão de Poisson. Não se utilizou a regressão logística porque esta teria, como desfecho, a ocorrência de uma ou mais injúrias, não levando em conta a repetição das injúrias nas crianças. Estudos anteriores utilizaram a regressão de Poisson para análises de taxas de ocorrência de injúrias acidentais ${ }^{18-20}$. Este tipo de análise permite ainda o ajuste de diversas covariáveis ${ }^{19}$. 
Tabela 4 - Análise de regressão de Poisson múltipla dos fatores de risco para injúrias acidentais, ajustadas para fatores de confusão, Pelotas, 1998

\begin{tabular}{|c|c|c|c|c|c|}
\hline & Variáveis & & Análise bruta & Análise ajustada & p valor (ajustado) \\
\hline \multirow[t]{6}{*}{ NÍVEL 1} & Cor da criança & $\begin{array}{l}\text { não branca } \\
\text { branca }\end{array}$ & $\begin{array}{c}1,0 \\
1,70(1,27-2,25)\end{array}$ & $\begin{array}{c}1,0 \\
1,70(1,28-2,25)\end{array}$ & $<0,001$ \\
\hline & Sexo & $\begin{array}{l}\text { feminino } \\
\text { masculino }\end{array}$ & $\begin{array}{c}1,0 \\
1,39(1,13-1,70)\end{array}$ & $\begin{array}{c}1,0 \\
1,32(1,08-1,63)\end{array}$ & $<0,001$ \\
\hline & Classe Social ABIPEME & $\begin{array}{l}\mathrm{A}+\mathrm{B} \\
\mathrm{C} \\
\mathrm{D} \\
\mathrm{E}\end{array}$ & $\begin{array}{c}1,0 \\
0,95(0,68-1,31) \\
0,84(0,61-1,16) \\
1,04(0,73-1,49)\end{array}$ & $\begin{array}{c}1,0 \\
1,13(0,79-1,58) \\
1,04(0,86-2,11) \\
0,84(0,61-1,14)\end{array}$ & 0,31 \\
\hline & $\begin{array}{l}\text { Renda Familiar } \\
\text { (salário-mínimo) }\end{array}$ & $\begin{array}{l}>6 \\
3,01-6,0 \\
1,01-3,0 \\
<1,0\end{array}$ & $\begin{array}{c}1,0 \\
0,77(0,58-1,06) \\
1,09(0,85-1,42) \\
0,78(0,55-1,12)\end{array}$ & $\begin{array}{c}1,0 \\
0,83(0,61-1,14) \\
1,21(0,89-1,65) \\
0,89(0,58-1,36)\end{array}$ & 0,22 \\
\hline & Trabalho materno & $\begin{array}{l}\text { não } \\
\text { sim }\end{array}$ & $\begin{array}{c}1,0 \\
0,90(0,73-1,11)\end{array}$ & $\begin{array}{c}1,0 \\
0,88(0,72-1,08)\end{array}$ & 0,31 \\
\hline & $\begin{array}{l}\text { Escolaridade materna } \\
\text { (anos) }\end{array}$ & $\begin{array}{l}\geq 9 \\
5-9 \\
0-4\end{array}$ & $\begin{array}{c}1,0 \\
0,80(0,64-1,04) \\
0,82(0,70-1,22)\end{array}$ & $\begin{array}{c}1,0 \\
0,76(0,58-1,01) \\
0,72(0,52-1,01)\end{array}$ & 0,12 \\
\hline \multirow[t]{6}{*}{ NÍVEL 2* } & Idade materna & $\begin{array}{l}<20 \\
20-29 \\
30-35 \\
>35\end{array}$ & $\begin{array}{c}1,0 \\
0,80(0,65-1,14) \\
0,95(0,67-1,34) \\
0,67(0,41-1,02)\end{array}$ & $\begin{array}{c}1,0 \\
0,87(0,66-1,14) \\
0,99(0,70-1,39) \\
0,78(0,47-1,23)\end{array}$ & 0,60 \\
\hline & Paridade & $\begin{array}{l}<4 \\
\geq 4\end{array}$ & $\begin{array}{c}1,0 \\
1,51(0,98-2,32)\end{array}$ & $\begin{array}{c}1,0 \\
1,52(0,99-2,34)\end{array}$ & 0,16 \\
\hline & Tipo de casa & $\begin{array}{l}\text { outros materiais } \\
\text { tijolo }\end{array}$ & $\begin{array}{c}1,0 \\
1,36(1,02-1,82)\end{array}$ & $\begin{array}{c}1,0 \\
1,39(1,03-1,88)\end{array}$ & 0,03 \\
\hline & Irmão menor na casa & $\begin{array}{l}\text { não } \\
\text { sim }\end{array}$ & $\begin{array}{c}1,0 \\
1,26(1,02-1,55)\end{array}$ & $\begin{array}{c}1,0 \\
1,34(1,08-1,67)\end{array}$ & 0,04 \\
\hline & Mora no centro & $\begin{array}{l}\operatorname{sim} \\
\text { não }\end{array}$ & $\begin{array}{c}1,0 \\
1,37(1,11-1,80)\end{array}$ & $\begin{array}{c}1,0 \\
1,33(0,98-1,80)\end{array}$ & 0,07 \\
\hline & Aglomeração & $\begin{array}{l}2-3 \\
4-5 \\
>5\end{array}$ & $\begin{array}{c}1,0 \\
0,83(0,66-1,05) \\
1,14(0,81-1,60)\end{array}$ & $\begin{array}{c}1,0 \\
0,86(0,68-1,08) \\
1,16(0,84-1,60)\end{array}$ & 0,14 \\
\hline \multirow[t]{2}{*}{ 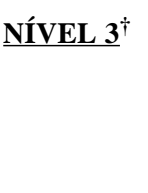 } & Idade gestacional & $\begin{array}{l}\geq 37 \\
<37\end{array}$ & $\begin{array}{c}1,0 \\
1,14(0,81-1,62)\end{array}$ & $\begin{array}{c}1,0 \\
1,22(0,84-1,75)\end{array}$ & 0,33 \\
\hline & Peso de nascimento & $\begin{array}{l}<2500 \\
\geq 2500\end{array}$ & $\begin{array}{c}1,0 \\
1,43(0,97-2,12)\end{array}$ & $\begin{array}{c}1,0 \\
1,46(0,96-2,23)\end{array}$ & 0,07 \\
\hline \multirow[t]{4}{*}{ 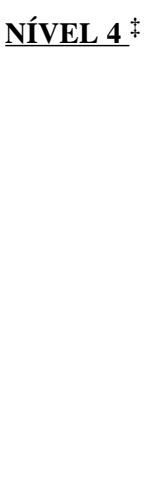 } & Quem cuida da criança & $\begin{array}{l}\text { outro } \\
\text { mãe ou pai }\end{array}$ & $\begin{array}{c}1,0 \\
1,15(0,93-1,42)\end{array}$ & $\begin{array}{c}1,0 \\
1,35(0,99-1,82)\end{array}$ & 0,07 \\
\hline & $\begin{array}{l}\text { Teste de triagem de } \\
\text { desenvolvimento }\end{array}$ & $\begin{array}{l}\text { negativo } \\
\text { positivo }\end{array}$ & $\begin{array}{c}1,0 \\
0,87(0,70-1,08)\end{array}$ & $\begin{array}{c}1,0 \\
0,90(0,70-1,11)\end{array}$ & 0,31 \\
\hline & Duração da amamentação & $\begin{array}{l}\text { nunca mamou } \\
<3 \mathrm{~m} \\
3 \text { a } 6 \mathrm{~m} \\
6 \text { a } 12 \mathrm{~m} \\
>12 \mathrm{~m}\end{array}$ & $\begin{array}{c}1,0 \\
1,20(0,79-1,80) \\
1,02(0,96-2,28) \\
1,47(0,96-2,28) \\
1,12(0,73-1,73)\end{array}$ & $\begin{array}{c}1,0 \\
1,10(0,65-1,88) \\
0,90(0,51-1,63) \\
1,72(0,96-3,03) \\
1,15(0,66-2,01)\end{array}$ & 0,26 \\
\hline & $\begin{array}{l}\text { Freqüenta creche } \\
\text { ou escola }\end{array}$ & $\begin{array}{l}\text { não } \\
\text { sim }\end{array}$ & $\begin{array}{c}1,0 \\
1,14(0,88-1,46)\end{array}$ & $\begin{array}{c}1,0 \\
1,28(0,94-1,77)\end{array}$ & 0,10 \\
\hline
\end{tabular}

* Nível 2 - ajustado para cor, sexo, renda e escolaridade da mãe; † Nível 3 - ajustado para cor, sexo, renda familiar, escolaridade da mãe, idade da mãe, tipo de construção, presença de irmão menor na casa e morar no centro ou não; ¥ Nível 4 - ajustado para as variáveis anteriores e para peso de nascimento e idade gestacional 
Na interpretação dos resultados da análise acima mencionada, é importante considerar a relação hierárquica entre os fatores de risco em estudo. $\mathrm{O}$ efeito dos fatores socioeconômicos é mediado por outros fatores, como os reprodutivos e os ambientais. A análise realizada levou em conta esta hierarquia.

A incidência de injúrias acidentais foi maior entre os meninos em relação às meninas. A maioria dos estudos confirma estes achados, sugerindo que o maior acometimento esteja relacionado à exposição dos meninos aos agentes e situações de risco ${ }^{5,6,10,18,20}$.

Crianças de cor branca apresentaram incidência de acidentes $70 \%$ superior às demais crianças. Este aumento na taxa não foi detectado em estudo britânico ${ }^{6}$. Uma possível explicação para este achado seria que a cor da criança estivesse atuando como um marcador da situação socioeconômica, mas, mesmo após ajuste para possíveis fatores mediadores como renda e escolaridade, a RDI permaneceu inalterada. Não se pode excluir a possibilidade de viés de informação, isto é, que famílias brancas houvessem relatado um maior número de injúrias, apesar de a real incidência ter sido similar a das crianças não-brancas. Sabendo-se as dificuldades de definir-se a variável cor, utilizou-se como referência a cor observada pelo entrevistador, apesar de em outro estudo pelotense recente ter-se constatado que o kappa para concordância entre a cor referida pelo sujeito e a cor observada foi de 0,84 (C. Victora, comunicação pessoal).

Não se encontraram associações significativas entre a ocorrência de injúrias acidentais e a renda familiar ou a escolaridade paterna ou materna. Esses dados são controversos na literatura: alguns estudos indicam maior ocorrência de acidentes em crianças com situação econômica mais favorável ${ }^{10,11}$, embora outros contrariem essa informação ${ }^{6,20}$. A maioria desses estudos referem-se apenas às lesões que tiveram assistência médica. No presente estudo, porém, foram referidas inclusive as lesões menos graves sofridas pela criança, tendo sido evitado, portanto, um possível viés de procura de assistência médica entre as famílias mais ricas.

Alguns estudos têm encontrado associação entre trabalho materno e ocorrência de injúrias acidentais ${ }^{21}$. No presente trabalho, porém, não se encontrou associação significativa $(\mathrm{p}=0,32)$.

$\mathrm{Na}$ análise, foi observado que a idade materna inferior a 20 anos esteve aparentemente associada à maior incidência de injúrias acidentais, mas, após controle para variáveis de confusão, este efeito não permaneceu. Hijar-Medina et al., no entanto, observaram esse efeito, mesmo após ajus$\mathrm{te}^{22}$.

Tanto na análise bruta quanto na ajustada, aquelas crianças com irmãos menores residindo na casa apresentavam taxas maiores de lesão. Hijar-Medina et al. ${ }^{22}$ confirmaram este efeito, em seu estudo de casos e controles para acidentes domésticos, em crianças com idade inferior a cinco anos. Acredita-se que, nesta faixa etária, a criança ainda demande uma atenção especial por parte de seus pais ou pessoa responsável pelo cuidado. No momento em que a criança tem um irmão menor, as atenções tendem a voltarse para este irmão. É importante ainda levar em conta o estágio do desenvolvimento infantil. As crianças estudadas eram pré-escolares, que apresentam um tipo de pensamento mágico, com percepção egocêntrica e com uma lógica própria de interpretar seu ambiente, não sendo capazes, ainda, de aprender noções de segurança ${ }^{23}$. Como conseqüência, no momento em que passa a ser o irmão "mais velho", em decorrência de possuir um "mais novo", é muitas vezes o responsável por seu "autocuidado", portanto, mais suscetível de sofrer injúrias acidentais.

Crianças que moram em casas de tijolo tiveram maior risco de se lesionarem do que as que moram em casas de outros materiais. Não parece existir nenhuma razão evidente para esse achado. É possível que a moradia em casas de tijolo seja um marcador de nível socioeconômico e que esteja associado a um viés de informação, ou seja, essas famílias tenderiam a notificar melhor os acidentes. Por outro lado, crianças residentes em zonas periféricas da cidade apresentaram tendência a sofrerem mais lesões $(\mathrm{p}=0,07)$. Possível explicação para este fato é de que essas crianças estejam expostas a maiores riscos ambientais, pois as da zona central ficam confinadas ao seu ambiente. Já as crianças de periferia permanecem mais tempo na rua, com maior exposição à ocorrência de injúrias acidentais. Não se encontrou na literatura nenhuma referência sobre os efeitos do tipo de construção. Quanto ao local de moradia, sabe-se que os riscos de lesões físicas em crianças, em zonas urbanas e rurais, diferem muito entre si. Geralmente, em áreas rurais, há menor acesso a serviços de saúde, aumentando, principalmente, a taxa de mortalidade decorrente das injúrias acidentais ${ }^{24}$. Addor et al., entretanto, quando estudaram a incidência de injúrias acidentais entre pré-escolares na Suíça, verificaram que não havia diferença levandose em conta o local de moradia (urbano ou rural) ${ }^{11}$.

Crianças com peso de nascimento superior a 2.500 gramas tiveram uma taxa aumentada de injúrias acidentais. Hack et al. ${ }^{25}$, em seu estudo prospectivo sobre a saúde de crianças de muito baixo peso, realizado em um período de oito anos, encontraram taxas de acidentes similares para crianças de muito baixo peso e as de peso normal. Por outro lado, no estudo de coorte das crianças nascidas em 1982, em Pelotas, RS, crianças com peso de nascimento superior a 2.500 gramas tiveram uma taxa maior de fraturas ${ }^{26}$.

Vários estudos têm demonstrado ser a creche um ambiente seguro para as crianças ${ }^{27,28}$. No presente estudo, apenas três por cento de todas as lesões ocorreram na escola ou na creche e, mesmo entre as 107 crianças que as freqüentavam, apenas $14,7 \%$ dos acidentes relatados ocorreram nesses locais. Na análise ajustada, a associação entre freqüência à creche ou à escola e a incidência de lesões apresentou um nível de significância de $10 \%$.

Este estudo permitiu detectar os principais fatores de risco para ocorrência de injúrias acidentais em nosso meio. 
Além disso, foi o primeiro estudo prospectivo, realizado no Brasil, que levou em consideração não apenas a ocorrência de injúrias acidentais, mas também a diferença existente entre o número de lesões sofridas pela criança. A partir destes dados, podem-se conhecer melhor os grupos de risco em nosso meio. Os fatores de risco identificados podem ser divididos em três grupos. O primeiro grupo inclui o sexo masculino e a presença de irmãos menores. Esses dois fatores apresentam plausibilidade biológica e encontrou-se uma associação significativa com o desfecho. $\mathrm{O}$ segundo grupo (peso de nascimento $>2.500$ gramas, freqüência à creche ou escola e residência na periferia urbana) também apresenta plausibilidade biológica, mas a associação estatística com as injúrias acidentais foi limítrofe (p entre 0,05 e 0,10$)$. Por fim, não foi possível encontrar explicação causal plausível para as associações significativas com a cor branca, moradia em casa de tijolos e o fato de ser cuidado pelos pais. Resta, portanto, a possibilidade de que essas associações sejam devidas a viés de informação. Destes fatores, a presença de crianças mais jovens em casa merece especial atenção, sendo necessário instruir os pais sobre o aumento no risco observado por ocasião do nascimento de um irmão menor. Esses dados podem ser úteis para promoção de campanhas de esclarecimento aos pais e à sociedade em geral, incentivando a realização de novas pesquisas semelhantes em outros locais.

\section{Referências bibliográficas}

1. Gofin R, Lison M, Morag C. Injuries in primary care practices. Arch Dis Child 1993; 68:223-6.

2. Rodriguez JG, Brown ST. Childhood injuries in the United States. AJDC 1990; 144(6):625-6.

3. Irvin CE, Cataldo MF, Matheny AP, Peterson L. Health consequences of behaviors: injury as a model. Pediatrics 1992;90(5): 798-807.

4. Fonseca SS. Distribuição e características dos acidentes em crianças de Pelotas, RS, Brasil: comparação de duas metodologias [dissertação]. Pelotas: Universidade Federal de Pelotas; 1999.

5. Bussing R, Menvielle E, Zima B. Relationship between behavioral problems and unintentional injuries in US children. Arch Pediatr Adolesc Med 1996;150(1):50-6.

6. Alwash R, Mccarthy M. Accidents in the home among children under 5: ethnic differences or social disadvantage? BMJ 1988;296(5):1450-3.

7. Unglert CV, Siqueira A, Carvalho GA. Características epidemiológicas dos acidentes na infância. Rev Saúde Pública 1987;21(3): 224-45.

8. Bangdiwala SI, Anzola-Perez E, Romer CC, Schimidt B, Valdez-Lazo F, Toro J, et al. The incidence of injuries in young people: I methodology and results of a collaborative study in Brazil, Chile, Cuba and Venezuela. Int J Epidemiol 1990;19(1): 115-24.

9. Mathire DN, Nsung M, Mabhiza ET. Factors associated with incidence of domestic accidents in children aged 0-5 years in Chikomba District, Mashonaland East, Zimbabwe. Cent Afr J Med 1994;40(5):113-9.

10. Teixeira AMFB. Morbidade por acidentes em menores de dez anos, em Pelotas, RS: prevalência e caracterização dos acidentes. Pelotas, 1992 [dissertação]. Pelotas: Universidade Federal de Pelotas; 1992.
11. Addor V, Eggimann BS. Population-based incidence of injuries among preschoolers. Eur J Pediatr 1996;155:130-5.

12. Victora CG, Barros FC, Halpern R, Menezes AM, Horta BL, Tomasi E, et al. Estudo longitudinal da população maternoinfantil de Pelotas, RS, 1993: aspectos metodológicos e resultados preliminares 1996. Rev Saúde Pública 1997;30:34-45.

13. Dubowitz L, Dubowitz V, Goldeberg C. Clinical assessment of gestational age in the newborn infant. Pediatrics 1970;77:1.

14. Frankenburg KW, Doodds J, Archer P, Bresnick B. Denver II: Technical manual and training manual. Denver: Denver Developmental Material Inc; 1990.

15. Victora CG, Huttly SR, Fuchs SC, Olinto MT. The role of conceptual frameworks in epidemiological analysis: a hierarchical approach. Int J Epidemiol 1997;26(1):224-7.

16. Barros AJD. Acute lower respiratory infection among children in The Gambia. An evaluation of risk factors with emphasis on nutritional status [dissertation]. London: London School of Hygiene and Tropical Medicine; 1991.

17. Agresti A. An introduction to categorical data analysis. The Generalized Models 1996; chapter 4:92-3.

18. Roberts I, Power C. Does the decline in child injury mortality vary by social class? A comparison of class specific mortality in 1981 and 1991. BMJ 1996;313(7060):784-6.

19. Kuhn L, Davidson LL, Durkin MS. Use of Poisson regression and time series analysis for detecting changes over time in rates of child injury following a prevention program. Am J Epidemiol 1994;140(10):943-55.

20. Anderson CL, Agran PF, Winn DG, Tran C. Demographic risk factors for injury among Hispanic and non-Hispanic white children: an ecologic analysis. Inj Prev 1998;4(1):33-8.

21. Hijar-Medina, Tapia-Yáñez JR, López-lópez MV. El trabajo materno y la gravedad de lesiones accidentales en niños. Salud Pública de Mex 1995;37(3):198-204.

22. Hijar-Medina, Tapia-Yáñez JR, López-lópez MV, Flores-Solórzano LI, Ascencio-Lozano R. Factores de riesgo de accidentes en el hogar en niños. Estudio de casos y controles. Bol Med Hosp Infant Mex 1993;50(7):463-74.

23. Blank D. Conceitos básicos e aspectos preventivos gerais. In: Blank D, ed. Manual de Acidentes e Intoxicações na Infância e Adolescência. Rio de Janeiro: SBP; 1994. p.2-13.

24. Hui-Chia Hwang, Stallones L, Keefe J. Childhood injury deaths: rural and urban differences, Colorado 1980-8. Inj Prev 1996; 2:105-8.

25. Hack M, Weissman B, Breslau N, Klein N, Fanaroff AA. Health of very low birth weight children during their first eight years. J Pediatr 1993;122:887-92.

26. Victora CG, Barros FC, Vaughan JP. Epidemiologia da desigualdade. Hospitalizações e doenças. São Paulo: Ed. Hucitec; 1988. p.86-93.

27. Kotch JB, Dufort VM, Stewart P, Fieberg J, McMurray M, $\mathrm{O}$ 'Brien S, et al. Injuries among children in home and out-ofhome care. Inj Prev 1997;3:267-71.

28. Cummings P, Rivara FP, Boase J, MacDonald JK. Injuries and their relation to potential hazards in child day care. Inj Prev 1996;2:105-8.

Endereço para correspondência:

Dra. Sílvia Stringari Fonseca

Hospital Escola- UFPEL

Rua Professor Araújo, 538 - Pelotas, RS

Fone: 53227.7944 - ramal 246

E-mail: silviasf@terra.com.br 